\title{
Experiencia en 10 años de aplicación de fibrobroncoscopia en pacientes pediátricos
}

\author{
Ignacio Sánchez D, Caterina Pesce $A^{a}$, Héctor $N$ avarro $M^{b}$, \\ Linus H olmgren P, Pablo Bertrand N, Cecilia Alvarez G.
}

\section{A ten years experience in flexible bronchoscopy in pediatric patients}

Background: Approximately a decade ago, pediatric Flexible Bronchoscopy (FB) was introduced in Chile, after being used for several years in adults. Aim: To describe our clinical experience in FB in a ten years period. Patients and Methods: Records of procedures done between January 1993 and September 2002 at the Pediatric Service of the Catholic University Hospital, were retrospectively reviewed. We evaluated the clinical indications for the procedures in relation to patient's age and the correlation between indications and FB findings. Results: A total of 700 procedures were performed during the period, $59 \%$ in men and $53 \%$ in patients younger than 1 year. Seventy seven percent of procedures were done in an examination room, using a nasal approach. The main indication was visualization of the airway (49\%). The most common clinical diagnosis, in descending order were: atelectasis, stridor and etiologic study of pneumonia by bronchoalveolar lavage (BAL). In children younger than 6 months the most common clinical diagnosis was stridor, followed by atelectasis. The main diagnosis in the whole sample, reached by FB was atelectasis secondary to mucous plug. In children younger than 6 months, the main diagnosis was laryngomalacia. A positive microbial culture was obtained in $43 \%$ of patients in whom BAL was done. Complications were uncommon (5\%) and mostly mild. In $2.3 \%$ of cases, these were severe, such as bronchospasm and need for mechanical ventilation. Severe complications were observed in patients younger than 3 months with severe stridor or in children with cancer, who required FB and BAL Conclusions: Flexible bronchoscopy is a safe and useful procedure in pediatric patients (Rev Méd Chile 2003; 131: 1266-72).

(Key Words: Atelectasis; Bronchoalveolar lavage; Bronchoscopy; Pneumonia)

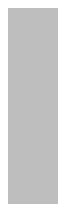

Recibido el 4 de abril, 2003. Aceptado en versión corregida el 4 de agosto, 2003.

Sección Respiratorio Pediátrico, Departamento de Pediatría, Pontificia Universidad Católica de Chile.

a Interna de Medicina, Pontificia Universidad Católica de Chile.

b Becario del Programa de Beca en Enfermedades Respiratorias del Niño. Pontificia Universidad Católica de Chile.

Correspondencia a: Dr. Ignacio Sánchez D. Sección de Respiratorio Pediátrico, Departamento de Pediatría, Pontificia Universidad Católica de Chile. Lira 44 - 1er piso. Santiago, Chile. Fono: 354 3887. Fax: 6384307.

E mail: igsan@med.puc.cl 
L a fibrobroncoscopia (FBC) pediátrica ha sido utilizada en el mundo desde hace más de 20 años; en este período se han publicado sus indicaciones, técnica, utilidad y complicaciones ${ }^{1-5}$. Así también, han habido varias publicaciones que han comparado las indicaciones del procedimiento flexible versus rígido, siendo de elección el primero en la visualización dinámica de la vía aérea, y en los procedimientos que no requieren sedación $^{6-9}$. Las principales indicaciones del procedimiento con broncoscopia rígida se relacionan con la extracción de un cuerpo extraño y otros procedimientos más invasivos ${ }^{10-12}$. La gran mayonía de las publicaciones provienen de países desarrollados, dentro de los que destaca Estados Unidos y los provenientes de Europa e Israel. En nuestro país esta técnica se introdujo hace 10 años, al comienzo se realizó en un grupo reducido de centros de la especialidad, para en los últimos años hacerse más extendida a múltiples lugares tanto en Santiago como en provincias. Desde el punto de vista de las publicaciones, en la literatura nacional destaca una experiencia tabulada y la presentación de series de magnitud variable en diversos congresos de la especialidad.

En las series extranjeras publicadas, destaca que desde su uso masivo, el procedimiento ha tenido una gran utilidad en el estudio de las patologías de la vía aérea superior e inferior, como también en el estudio etiológico de los cuadros infecciosos en pacientes inmunocompetentes $y$, en especial, en los que presentan trastornos en su sistema inmunitario ${ }^{13-15}$. En los últimos años, también se han desarrollado nuevas indicaciones para realizar procedimientos invasivos a través de la FBC, como son el sellamiento de fístulas traqueoesofágicas, broncopleurales, en algunos casos extracción de cuerpo extraño, instalación de estabilizadores de la vía aérea y otros ${ }^{11,13,16}$.

El objetivo del presente trabajo fue describir nuestra experiencia clínica en fibrobroncoscopia pediátrica en un período de 10 años, describiendo las indicaciones, población estudiada, hallazgos clínicos y complicaciones.

\section{PACIENTES Y MÉTODO}

En forma retrospectiva se incluyeron los pacientes que tuvieron procedimiento de fibrobroncoscopia realizados entre enero de 1993 y septiembre de 2002 en el Servicio de Pediatría del Hospital Clínico de la Universidad Católica de Chile.

En el estudio se utilizó un instrumento Olympus (BFC 3.6 y BF 3.1), de 3,5 mm de diámetro externo, con canal de succión de 1,2 mm y video cámara Sony. Los exámenes se realizaron en una sala de procedimientos especialmente acondicionada en el Servicio de Pediatría, en la Unidad de Cuidados Intensivos Pediátricos o en el pabellón de cirugía, de acuerdo a las condiciones generales y gravedad del paciente.

El paciente recibió sedación con atropina $(0,01$ $\mathrm{mg} / \mathrm{kg})$, morfina $(0,01 \mathrm{mg} / \mathrm{kg})$ y midazolam $(0,01$ $\mathrm{mg} / \mathrm{kg}$ ), en las dosis necesarias para obtener una sedación suficiente. En forma excepcional, se utilizó propofol o ketamina. En todos los pacientes se realizó monitorización de saturación arterial de oxígeno $\left(\mathrm{SaO}_{2}\right)$ y frecuencia cardíaca, y en los casos de pacientes inestables, se utilizó monitor cardiorrespiratorio y presión arterial frecuente.

Se revisaron las fichas clínicas de los pacientes y la ficha realizada posterior a cada procedimiento, en la que se incluían los datos generales, indicación del estudio, patología asociada, tipo de sedación utilizada y hallazgos del examen. Junto a lo anterior, se evaluó las indicaciones en relación a la edad del paciente, la correlación entre las indicaciones y los hallazgos encontrados.

\section{RESULTADOS}

Durante el período de tiempo descrito, se realizaron 700 procedimientos, 410 (59\%) en pacientes masculinos y 290 (41\%) femeninos. La mayoría de los estudios (53\%) se realizaron en lactantes menores de 1 año, hospitalizados en sala por al menos 24 h (70\%); 77\% se realizó en una sala de procedimientos, y a través de la vía nasal (77\%) (Tabla 1). En el grupo de menores de 6 meses, el rango de edad fue de 2 días a 5 meses, con un promedio de 3,1 \pm 1 meses ( $\bar{x} \pm$ DS). La principal indicación fue la visualización de la vía aérea (49\%); luego la realización de lavado bronquial, con instilación de suero fisiológico y reexpansión de atelectasias (36\%) (Figura 1). Los diagnósticos previos a la realización del examen fueron variados, siendo los principales: atelectasia, estridor y el estudio etiológico de las neumonías en este 
Tabla 1. Características generales de los pacientes

\begin{tabular}{|llrr|}
\hline & & $\mathrm{n}$ & $\%$ \\
\hline Sexo: & Hombres & 410 & 59 \\
& Mujeres & 290 & 41 \\
& $<6$ meses & 177 & 25 \\
& 6 meses - 1 año & 198 & 28 \\
Procedencia: & $1-5$ años & 171 & 25 \\
& $>5$ años & 154 & 22 \\
Lugar: & Ambulatorio & 95 & 14 \\
& UCI pediátrica & 115 & 16 \\
Vía: & Hospitalizados & 490 & 70 \\
& Sala de procedimientos & 540 & 77 \\
& UCI (pediátrica y neonatal) & 125 & 18 \\
& Pabellón cirugía & 35 & 5 \\
& Nasal & 540 & 77 \\
& Tubo endotraqueal & 72 & 10 \\
& Traqueostomía & 60 & 9 \\
& Máscara laríngea & 28 & 4 \\
\hline
\end{tabular}

Tabla 2. D iagnóstico previo a la realización de la fibrobroncoscopia. Comparación entre el grupo total y los pacientes menores de 6 meses

\begin{tabular}{|lrrrc|}
\hline & \multicolumn{2}{c}{ Grupo total } & \multicolumn{2}{c|}{$<6$ meses } \\
& $\mathrm{n}=700$ & $\%$ & $\mathrm{n}=177$ & $(\%$ \\
\hline Atelectasia & 242 & 35 & 43 & $24 *$ \\
Estridor & 144 & 21 & 68 & $38^{*}$ \\
Neumonía & 108 & 15 & 9 & $5 *$ \\
Tos crónica & 14 & 2 & 2 & 1 \\
Cuerpo extraño & 12 & 2 & 0 & 0 \\
Misceláneos & 180 & 26 & 55 & 31 \\
\hline
\end{tabular}

$* \mathrm{p}<0,05$.

Tabla 3. Tipo de patología asociada en los pacientes enviados a FBC

\begin{tabular}{|lrc|}
\hline Patología & $\mathrm{n}$ & $\%$ \\
\hline Genetopatías & 73 & 22 \\
DBP o DPCr* & 71 & 22 \\
Inmunodeficiencia & 38 & 12 \\
Enfermedad neuromuscular & 29 & 9 \\
Cardiopatía congénita & 60 & 18 \\
Otras & 54 & 17 \\
Total & 325 pacientes \\
\hline
\end{tabular}

*DBP o DPCr: Displasia broncopulmonar o daño pulmonar crónico. orden para la serie total, lo que varió en forma significativa al analizar el subgrupo de menores de 6 meses (prueba exacta de Fisher, $p<0,05$ ), (Tabla 2). Al considerar el tipo de patología asociada en los pacientes enviados a FBC, los principales diagnósticos fueron displasia broncopulmonar 0 daño pulmonar crónico y genopatías (Tabla 3).

Los resultados obtenidos en el estudio de la FBC fueron estadísticamente diferentes en la serie total, en que el principal diagnóstico fue la atelectasia secundaria a tapón mucoso, versus en el grupo de

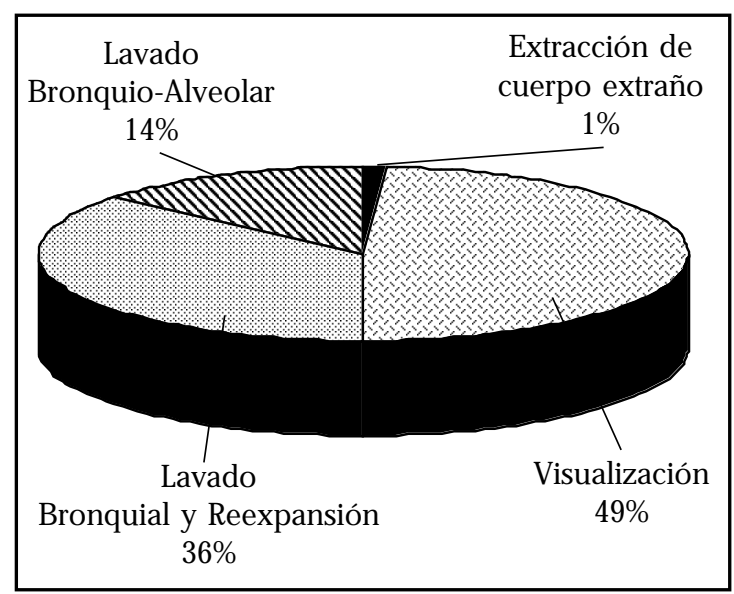

FIgURA 1. Indicaciones de realización de fibrobroncoscopia $(n=700)$. 
Tabla 4. Resultado obtenido en el estudio de FBC de la serie total $(n=700)$ y en el grupo $<6$ meses $(n=177)$

\begin{tabular}{|lrrrr|}
\hline Hallazgo & \multicolumn{2}{c}{ Serie Total } & \multicolumn{2}{c|}{ Grupo <6 meses } \\
& $\mathrm{n}=700$ & $\%$ & $\mathrm{n}=177$ & $\%$ \\
\hline Atelectasia (tapón mucoso) & 159 & 23 & 16 & $9 *$ \\
Laringomalacia & 134 & 19 & 85 & $48 *$ \\
Alteración obst. de la vía aérea superior & 76 & 11 & 15 & 8 \\
Malformación traqueobronquial & 77 & 11 & 15 & 8 \\
Normal & 76 & 11 & 10 & 6 \\
Traqueobroncomalacia & 58 & 8 & 23 & 13 \\
Misceláneo & 61 & 9 & 13 & 7 \\
IRA & 51 & 7 & 0 & 0 \\
Cuerpo extraño & 8 & 1 & 0 & 0 \\
\hline
\end{tabular}

$* \mathrm{p}<0,05$.

menores de 6 meses, en que el principal diagnóstico fue laringomalacia (prueba exacta de Fisher $p$ $<0,05$ ), (Tabla 4). Los pacientes que tuvieron FBC junto a lavado broncoalveolar (LBA) presentaron cultivos positivos en $43 \%$ de los casos, siendo de mayor utilidad en el grupo de inmunosuprimidos. Un completo análisis de esta serie se publicó recientemente por nuestro grupo ${ }^{17}$. Las Figuras 2 y 3 muestran el riesgo relativo de los diferentes diagnósticos encontrados en los pacientes que se estudiaron por estridor y atelectasia respectivamente.

Las complicaciones que se observaron en el estudio fueron principalmente episodios de desaturación y aumento de requerimientos de oxígeno con un bajo porcentaje de pacientes con conexión a ventilación mecánica (Tabla 5). Estas complicaciones se presentaron principalmente en dos grupos de pacientes: los menores de 3 meses portadores de obstrucción de la vía aérea superior, y pacientes mayores de 1 año, con patología oncológica de base, en quienes se requería realizar una FBC con lavado broncoalveolar para el estudio etiológico de los infiltrados pulmonares.

\section{Tabla 5. Complicaciones del estudio de fibrobroncoscopia}

\begin{tabular}{|lrc|}
\hline Complicación & $\mathrm{n}$ & $\%$ \\
\hline Sin complicación & 665 & 95 \\
Aumento requerimientos de $\mathrm{O}_{2}$ & 19 & 2,7 \\
Broncoespasmo & 5 & 0,7 \\
Conexión a ventilación mecánica & 11 & 1,6 \\
\hline
\end{tabular}

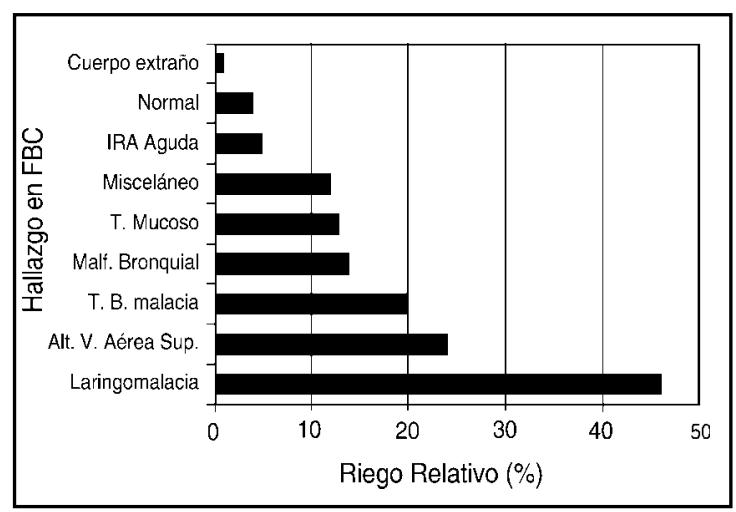

FIgURA 2. Resultado del estudio endoscópico de los pacientes con diagnóstico clínico de estridor $(n=144)$. Riesgo relativo se expresa en porcentaje.

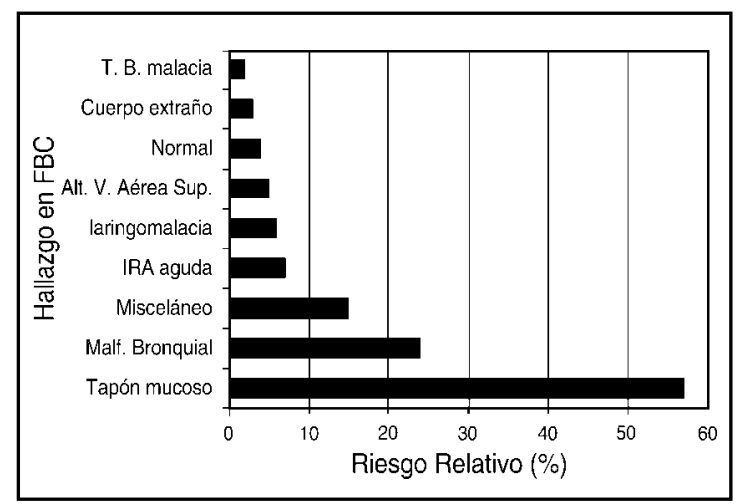

Figura 3. Resultado del estudio endoscópico de los pacientes con diagnóstico clínico de atelectasia $(n=242)$. Riesgo relativo se expresa en porcentaje. 


\section{DisCUSIÓN}

Nuestros resultados demostraron que las principales indicaciones diagnósticas para la FBC fueron atelectasia, estridor y el estudio etiológico de las neumonías, orden variable dependiendo de la edad del niño. En relación al resultado final del estudio, lo más frecuente fue laringomalacia en el menor de 6 meses, y atelectasia secundaria a tapón mucoso en la serie total. Las complicaciones fueron poco frecuentes y dependieron de la edad y de las condiciones generales del paciente. La gran mayoría de las publicaciones provienen de países desarrollados, destacando la ausencia de centros de Sudamérica.

Godfrey y cols, publicaron la utilidad de la broncoscopia flexible en niños, examinando 200 exámenes consecutivos ${ }^{18}$. Las principales indicaciones fueron respiración ruidosa (26\%), neumonía recurrente $(21 \%)$, neumonía en un paciente inmunosuprimido (10\%) y atelectasia (12,5\%). El resultado del estudio fue anormal en $67 \%$ de los casos, contribuyendo en la mayoría de los casos al manejo del paciente. Lo anterior se compara en forma cercana con nuestros hallazgos, describiéndose en nuestra serie un mayor número de malformaciones traqueobronquiales, y un porcentaje mayor de cultivos positivos en el lavado broncoalveolar ${ }^{17,29}$.

Gibson y cols, presentaron una sene de 35 fibrobroncoscopias en pacientes bajo los $10 \mathrm{~kg}$ de peso, 23 estudios orientados a visualizar la anatomía de la vía aérea, y 12 para LBA. El principal diagnóstico previo al examen fue estridor y atelectasia lobar, y la presencia de laningomalacia fue el principal hallazgo $^{19}$. Estos resultados los podemos comparar con los que observamos en nuestros pacientes menores de 6 meses, en que estridor fue la principal indicación, y el hallazgo más importante fue laringomalacia.

Pérez-Ruiz y cols, presentaron el análisis de una década en 536 fibrobroncoscopias en niños menores de 14 años $^{20}$. Las indicaciones más comunes fueron atelectasia persistente (31\%), estridor (25\%), tuberculosis (12\%), sospecha de cuerpo extraño (11\%) y sibilancias persistentes (10\%). La utilidad diagnóstica o terapéutica del procedimiento fue de $79 \%$. En nuestra serie, la principal intervención terapéutica fue la reexpansión de atelectasias (total o parcial), lo que se logró en $75 \%$ de los casos estudiados. La utilidad diagnóstica llegó a 89\%, lo que es incluso mayor, ya que un resultado normal puede descartar otras patologías ${ }^{27}$.

En un trabajo reciente, publicado en la literatura nacional, Prado y cols, analizaron un total de 137 FBC realizadas en un período de 2 años. En $70 \%$ de los casos el procedimiento fue diagnóstico, con las principales indicaciones: atelectasia (31\%), lavado broncoalveolar (22\%), estridor (14\%) y evaluación del paciente con traqueostomía (10\%). En el grupo de estridor congénito, la laringomalacia fue el diagnóstico principal (50\%), y el resultado diagnóstico del LBA fue de $25 \%$ de los casos. En esta serie se realizaron procedimientos que consistieron en extracción de cuerpo extraño, cierre de fístulas broncopleurales, intubación difícil y resección de un granuloma subglótico con el apoyo de broncoscopia rígida. Se reportó un porcentaje de complicaciones menor al $1 \% 21$. Schellbase y cols, publicaron la utilidad clínica de la broncoscopia flexible y LBA en lactantes con sibilancias recurrentes ${ }^{22}$, con un porcentaje significativo de LBA positivo a cultivos virales, y a macrófagos cargados de lípidos, sugerentes de aspiración pulmonar. Nuestra experiencia es menor, sin embargo hemos realizado estudios positivos en pacientes con hemosiderosis y reflujo gastroesofágico con aspiración pulmonar y macrófagos cargados con lípidos positivos.

Nussbaum y cols, publicaron la experiencia con el uso de la máscara laníngea en 92 pacientes, en particular en la reexpansión de atelectasias, estudio de infiltrados difusos y LBA en pacientes con tubo endotraqueal menor a $4 \mathrm{~mm}$. El procedimiento fue bien tolerado, no hubo presencia de complicaciones, con adecuada saturación de $\mathrm{O}_{2}$ en todos los pacientes ${ }^{23}$. Estos hallazgos son concordantes con los nuestros, en los pacientes en que se realizó el procedimiento con máscara laríngea, con un total de 28 casos y que han sido reportados en forma preliminar por Alvarez y $\operatorname{cols}^{24}$. Bibi y cols, publicaron la relación entre reflujo gastroesofágico y malacia en la vía aérea central $^{25}$. Los hallazgos fueron que en el grupo de estudio, 70\% tuvo RGE, comparado con 39\% en el grupo control encontrándose que el puntaje de macrófagos cargados con lípidos, se correlacionó con la certificación de RGE. En nuestra serie de traqueomalacia, la mayoría de los casos correspondió a pacientes con factores predisponentes, tales como cardiopatía congénita, daño neurológi- 
co y pulmonar crónico, y antecedentes de ventilación mecánica prolongada. No hubo antecedentes de RGE, sólo un paciente con historia de fístula traqueoesofágica operada, y sólo en 10\% de los casos se consideró el diagnóstico de traqueobroncomalacia primaria.

Barbato y cols, publicaron un catastro del uso de la broncoscopia pediátrica, flexible y rígida en 51 centros europeos, enviando cuestionario a los especialistas de los diferentes países, donde se preguntó por indicaciones, número de procedimientos realizados, tipo de sedación, LBA, efectividad diagnóstica y efectos adversos ${ }^{14}$. Cerca de $40 \%$ de los centros participaron en el estudio, reuniendo 4.587 broncoscopias flexibles, donde el principal diagnóstico fue neumonía recurrente o persistente en $17 \%$, seguidos de sibilancias que no responden a tratamiento $(15,5 \%)$, atelectasia persistente $(14 \%)$ y estridor (13\%). Nuestros resultados demuestran un bajo porcentaje de procedimientos realizados en pacientes con sibilancias sin respuesta a tratamiento, sin embargo los hallazgos de un grupo significativo de diagnóstico de traqueobroncomalacia, avala esta indicación. En cuanto al número de procedimientos, más de la mitad de los centros realizaban menos de 100 procedimientos al año, con sólo 10 hospitales en que se realizaban más de 200 broncoscopias al año. En nuestro centro, el promedio en el período de 10 años ha sido 70 estudios al año, lo que ha aumentado a valores cercanos a 100/año en los últimos 2 años. Con respecto a la sedación, la mayoría de los centros utilizaron midazolam y meperidina, existiendo alternativas como diazepam, ketamina y propofol en otros lugares. Por último, la utilidad del estudio en los pacientes en que se realizó broncoscopia flexible, alcanzó hasta $81 \%$ en el caso de estridor, seguida de atelectasia persistente (66\%), hemoptisis (66\%) y neumonía recurrente 0 persistente $(61 \%)$, utilidad similar a la encontrada en nuestro estudio.

La presencia de complicaciones y efectos adversos, en pacientes inmunocompetentes some-

\section{REFERENCIAS}

1. Green CG, Eisenberg J, Leong A, Nathanson I, Schnapf BM, Wood RE. Flexible endoscopy of the pediatric airway. Am Rev Respir Dis 1992; 145: 233-5. tidos a FBC fue estudiada por Picard y cols, en un grupo de 91 niños, de los cuales 48\% desarrolló fiebre dentro de las $24 \mathrm{~h}$ posterior a la broncoscopia, que no se asoció a bacteremia ${ }^{26}$. Los factores de riesgo para desarrollar esta complicación fueron la edad menor de 2 años, y el cultivo positivo en el LBA. Recientemente, de Blic y cols presentaron una revisión de 1.328 procedimientos, con complicaciones menores en 5,2\% (episodios transitorios de desaturación, tos excesiva, laringoespasmo transitorio) y $1,7 \%$ de complicaciones mayores, tales como desaturación moderada 0 severa, broncoespasmo, conexión a ventilación mecánica y pneumotórax ${ }^{16}$. Nuestras complicaciones fueron principalmente hipoxemia transitoria, que se corrigió con la administración de oxígeno, y en casos muy aislados obstrucción de la vía aérea superior, requiriendo intubación y ventilación mecánica, lo que se presentó en pacientes menores de 3 meses con estridor y en pacientes inmunosuprimidos con neumonía bilateral, a los que se les realizó el procedimiento para el estudio etiológico de su cuadro infeccioso.

En resumen, nuestro estudio presenta la experiencia de 10 años en la realización de estudios de fibrobroncoscopia en niños. El procedimiento tiene indicaciones precisas, las más frecuentes son el estudio de estridor, estudio y eventual tratamiento de la atelectasia secundaria a tapón mucoso, y el estudio etiológico de la neumonía complicada, o la que se presenta en pacientes inmunodeprimidos. Los hallazgos principales fueron laringomalacia y atelectasia debida a tapón mucoso ${ }^{27-29}$. Los pacientes estudiados con LBA tuvieron un cultivo positivo en $40 \%$ de los casos. Las complicaciones del estudio son poco frecuentes y en general no revisten mayor gravedad. Concluimos que la FBC es un procedimiento seguro y útil en el estudio de la vía aérea de los pacientes pediátricos. Nuestros resultados son muy similares a las publicaciones provenientes de países desarrollados.

2. Pérez CR, Wood RE. Update on pediatric flexible bronchoscopy. Pediatr Clin North Am 1994; 41: 385-400.

3. Wood RE. Pediatric bronchoscopy. Chest Surg Clin North Am 1996; 6: 237-51. 
4. Nicolai T. Pediatric bronchoscopy. Pediatr Pulmonol 2001; 31: 150-64.

5. De Blic J, Midula F, Barbato A, Ciement A, Dab I, EBER E ET AL. Bronchoalveolar lavage in children. ERS Task force on bronchoalveolar lavage in children. Eur Respir J 2000; 15: 217-31.

6. Mancuso RF. Stridor in neonates. Pediatr Clin North Am 1996; 43: 1339-56.

7. Riedier J, Grigg J, Robertson CF. Role of bronchoalveolar lavage in children with lung disease. Eur Respir J 1995; 8: 1725-30.

8. Cohn RC, Kenesmar C, Dearborn D. Safety and efficacy of flexible endoscopy in children with bronchopulmonary dysplasia. Am J Dis Child 1988; 142: 1225-8.

9. TAN I, SPARK LM. Flexible fiberoptic endoscopy for airway problems in a Pediatric Intensive Care Unit. Chest 1988; 93: 556-9.

10. Otte J, Girardi G, Contador AM. Diagnóstico y manejo del cuerpo extraño en la vía aérea. Rev Otorrinolaringol Cir Cabeza Cuello 1985; 2: 13-24.

11. Martinot A, Closset M, Marouette CH. Indications for flexible versus rigid bronchoscopy in children with suspected foreign body aspiration. Am J Respir Crit Care Med 1997; 155: 1676-9.

12. Swanson KL, Prakash UBS, Midthun DE, Edell ES, Utz JP, McDougall JC, Brutinel WM. Flexible bronchoscopy management of airway foreign bodies in children. Chest 2002; 121: 1695-700.

13. Nussbaum E. Pediatric fiberoptic bronchoscopy: clinical experience with 2.836 bronchoscopies. Pediatr Crit Care Med 2002; 3: 171-6.

14. Barbato A, Magarotto M, Crivallaro M, Navelo A Jr, Cracco A, De Buc J et al. Use of the paediatric bronchoscope, flexible and rigid, in 51 European centres. Eur Respir J 1997; 10: 1761-6.

15. Masters IB, Chang AB, Pattersoon L, Wainwright C, Buntain H, Dean BW, Francis PW. Series of laryngomalacia, tracheomalacia and bronchomalacia disorders and their associations with other condition in children. Pediatr Pulmonol 2002; 34: 189-95.

16. De Buc J, Marchac V, Scheinmann P. Complications of flexible bronchoscopy in children: prospective study of 1.328 procedures. Eur Respir J 2002; 20: 1271-6.

17. Zamorano A, Núñez A, Salnas ME, Navarro $H$, Holmgren L, Bertrand P et al. Experiencia clínica de la utilidad del lavado broncoalveolar en pediatría. Rev Chil Pediatr 2002; 73: 576-82.

18. Godfrey S, Avital A, MaAyan C, RotschiLd M, SPRINGER C. Yield from flexible bronchoscopy in children. Pediatr Pulmonol 1997; 23: 261-9.

19. Gibson NA, Coutts JAP, Patton JY. Flexible bronchoscopy under $10 \mathrm{~kg}$. Respiratory Medicine 1994; 88: 131-4.

20. Pérez-Ruiz E, Pérez-Farres J, Martínez-González B, Martínez-Aran T, Milano-Manso G, Martínez-ValVERDE A. Pediatric fiberoptic bronchoscopy. Analysis of a decade. An Esp Pediatr 2001; 55: 421-8.

21. Prado F, Boza ML, Badila JM, Isamitt D. Fibrobroncoscopia en pediatría: utilidad diagnóstica y terapéutica. Rev Chil Pediatr 1998; 69: 207-14.

22. ScheldaAse DE, Fawcett DD, Schutze GE, Lensing SY, TRYKE AF. Clinical utility of flexible bronchoscopy and bronchoalveolar lavage in young children with recurrent wheezing. J Pediatr 1998; 132: 312-8.

23. Nussbaum E, Zagnoev M. Pediatric fiberoptic bronchoscopy with a laryngeal mask airway. Chest 2001; 120: 614-6.

24. Alvarez C, Rodríguez JI, Ronco R, Castiшo A, Campos E, Sánchez I. Uso de mascarilla laríngea para fibrobroncoscopia en lactantes durante ventilación mecánica. Rev Chil Enf Respir 2002; 18: 103-11.

25. Bibi H, Khvols E, Shoseyor D, Ohaly M, Ben Dor D, LONDON D, ATER D. The prevalence of gastroesophageal reflux in children with tracheomalacia and laryngomalacia. Chest 2001; 119: 409-13.

26. Picard E, Schwartz S, Goldberg S, Glick T, Viшa Y, KEREM E. A prospective study of fever and bacteremia after flexible fiberoptic bronchoscopy in children. Chest 2000; 117: 573-7.

27. Holmgren NL, Córdova M, Ortúzar P, Sánchez I. Broncoscopia flexible en la reexpansión de atelectasias persistentes en pediatría. Arch Bronconeumol 2002; 38: 369-73.

28. Castiwo A, Smith J, Figueroa V, Bertrand P, Sánchez I. Traqueobroncomalacia en pacientes pediátricos: experiencia clínica. Rev Méd Chile 2002; 130: 1014-20.

29. Sánchez I, Navarro H, Méndez M, Holmgren N, Caussade S. Clinical characteristics of children with tracheobronchial anomalies. Pediatr Pulmonol 2003; 35: 288-91. 